\title{
Long-Term Survival, Toxicity Profile, and role of F-18 FDG PET/CT scan in Patients with Progressive Neuroendocrine Tumors Following Peptide Re- ceptor Radionuclide Therapy with High Activity In-111 Pentetreotide
}

\author{
Ebrahim S. Delpassand ${ }^{1,2}{ }^{\bowtie}$, Amin Samarghandi1 ${ }^{1}$, Jennifer Sims Mourtada ${ }^{2}$, Sara Zamanian², Gregory D. \\ Espenan ${ }^{3}$, Roozbeh Sharifi, Shawn MacKenzie ${ }^{4}$, Kambiz Kosari', Omar Barakat5, Shagufta Naqvi ${ }^{6}$, John E. \\ Seng ${ }^{7}$, Lowell Anthony8 \\ 1. Excel Diagnostics and Nuclear Oncology Center, 9701 Richmond Avenue, suite 122, Houston, Texas, 77042; \\ 2. RadioMedix, Inc. 9701 Richmond Avenue, suite 222 Houston, Texas, 77042; \\ 3. Physics Services Incorporated, 5000 West Esplanade, PMB 322, Metairie, LA 70006; \\ 4. Virginia Piper Cancer Institute, Liver and Pancreas Clinic, 800 East 28th Street, Suite 105, Minneapolis, MN 5407; \\ 5. St. Luke's Episcopal Hospital, 6720 Bertner Ave. Houston, TX 77030; \\ 6. Premier Oncology Consultants, 12121 Richmond Ave, Suite 208, Houston Texas, 77082; \\ 7. Minnesota Oncology, 910 Medical Place, 910 E 26th Street, Suite 200, Minneapolis, MN 55404; \\ 8. University of Kentucky in Lexington, KY.
}

Corresponding author: edelpassand@exceldiagnostics.com.

(C) Ivyspring International Publisher. This is an open-access article distributed under the terms of the Creative Commons License (http://creativecommons.org/ licenses/by-nc-nd/3.0/). Reproduction is permitted for personal, noncommercial use, provided that the article is in whole, unmodified, and properly cited.

Received: 2011.11.01; Accepted: 2012.01.01; Published: 2012.05.11

\begin{abstract}
Aim: To study the long term benefits, toxicity and survival rate in patients with neuroendocrine tumors receiving multiple cycles of high activity In-III Pentetreotide therapy. Moreover, our secondary aim was to evaluate the value of F-I8 FDG PET-CT scan as prognostic indicator in this group of patients.

Background: Neuroendocrine tumors are a heterogeneous group of malignancies which are usually metastatic at diagnosis. Standard chemotherapy in these patients is associated with appreciable adverse events and low effectiveness. Since 1990s, Peptide receptor radionuclide therapy (PRRT) with radio-labeled somatostatin analogues has been introduced as a new method of treatment in patients with unresectable and/or metastatic neuroendocrine tumors expressing high levels of Somatostatin receptors.

Methods: II 2 patients with progressive disseminated and unresectable neuroendocrine tumor (stage III and stage IV) were enrolled in a non-randomized trial in an out-patient setting. High activity In-I II Pentetreotide $(500 \mathrm{mCi}($ I8.5 GBq) per cycle) was administered as an intravenous infusion over 3 hours and repeated therapy cycles every 9-12 weeks in eligible patients up to maximum of 4 cycles. Response to therapy was evaluated by clinical imaging using the RECIST criteria, metabolic criteria and patient's quality of life questionnaire. Dosimetry and biodistribution studies were also performed. Finally, Kaplan-Meier survival analysis was performed for patients followed for greater than 12 months. The relationship between pretreatment F-I8 FDG PET-CT scan status and survival was determined by two-tailed Student's t-test in 42 patients who underwent pre-therapy PET scans.

Results: For an average of 25 (median 18.65) months following the therapy, patients were evaluated for any evidence of toxicity. No significant acute toxicity was observed in patients. Grade II or III hematological toxicity (7.6\% of patients), liver toxicity $(18.4 \%)$ and also grade I renal toxicity $(6.1 \%)$ was observed in 92 evaluable patients. Radiological responses were evaluated for an average of 29 months following their last cycle of therapy and results were
\end{abstract}


analyzed by the RECIST criteria. Majority (85\%) of patients had stable disease (SD), partial response (PR) rate was $7.5 \%$ and progressive disease (PD) was observed in $7.5 \%$ of patients. The average survival was 24.67 months after 2 cycles of therapy, 30.53 months after 3 cycles of therapy and 30.19 months after 4 cycles of therapy. Of the 42 patients who had pretreatment PET-CT imaging, 3I patients had positive F-I8 FDG scans $(S U V>2.5)$ with an average survival time of 18.9 months (range I.4-45.8 months) and II patients had negative F-I8 FDG scans (SUV $\leq 2.5$ ) with an average survival time of 31.8 months (range 7.4-42.9 months). Survival times for FDG negative patients were significantly longer than those for FDG positive patients ( $p=0.00 \mathrm{I}$ with $95 \%$ confidence).

Conclusion: High activity In-III therapy is a safe and effective therapy for patients with progressive disseminated neuroendocrine tumors. No major hematological, renal and hepatic toxicities were observed. There was an increase in survival time particularly in patients with lower degree of liver involvement as well as patients who received three or more cycles of therapy, as compared to historical data. Pre-treatment FDG status may be a predictor of survival following In-I I I pentetreotide therapy.

Key words: In-111 Pentetreotide therapy, neuroendocrine tumors, long term benefits, toxicity and survival rate, F-18 FDG PET/CT scan

\section{Introduction}

Neuroendocrine tumors (NET) are a rare, heterogeneous group of malignancies ${ }^{1,2}$. Typically, this category of malignancies are difficult to diagnose and treat ${ }^{3}$. While surgery can sometimes provide a curative treatment for patients with localized disease, NETs often present as late stage tumors with advanced metastatic disease ${ }^{3}$, making surgical resection impossible. Traditional chemotherapy and radiation treatment regimens may not be beneficial for patients with disseminated NETs.

A common feature of differentiated NETs is a high level expression of somatostatin receptors on their cell surface. This feature has been exploited by the use of radiolabeled somatostain analogues as a tool for diagnosis and treatment for NETs ${ }^{4}$. Scintigraphy with indium-111 labeled somatostatin analogue pentetreotide is a common method of detection of somatostatin receptor positive NETs. Ga-68 labeled somatostatin analogues have also been used for PET-CT scan in these patients recently ${ }^{5}$.

Indium-111 is a gamma emitter that also emits Auger and conversion electrons during its decay cycle. These electrons emit high energy at close range and are capable of inducing cytotoxic DNA damage ${ }^{6}$. Peptide receptor radionuclide therapy (PRRT) using somatostatin analogues labeled with high activity In-111 pentetreotide has been reported to be a safe and effective therapy for NETs ${ }^{7,8}$.

The first clinical trial of In-111 pentetreotide for the treatment of NETs was performed by Krenning et al. 7 in Netherlands in 1994. Preliminary data from this study demonstrated the safety of repeated treatments with $90-180 \mathrm{mCi}$ (3.3-6.6 GBq) of In-111 pentetreotide administered every 3 weeks for 10 cycles. In this ini- tial study, response correlated with receptor expression. Subsequent studies conducted by the same group confirmed these preliminary findings ${ }^{8}$. Likewise, additional investigational studies have reported significant responses to high-activity In-111 pentetreotide therapy (180-500 $\mathrm{mCi}$ (6.6-18.5 GBq) /treatment) with limited toxicity ${ }^{7-15}$. These studies vary in patient selection, dosing, total activity administered, and reported outcomes, but all show significant benefits and safety of In-111 pentetreotide therapy.

Generally, studies using higher activity of In-111 (180-500 mCi (6.6-18.5 GBq)) report a greater impact on disease stabilization than those using lower activity while still not reaching the maximum-tolerated dose (MTD). We previously reported the safety and efficacy of high activity In-111 pentetreotide therapy in 32 patients who were treated with one or two cycles of $500 \mathrm{mCi}(18.5 \mathrm{GBq})$ activity. The majority of these patients demonstrated disease stabilization and symptomatic improvement, with minimal hematologic toxicity. No evidence of long-term renal toxicity was reported in patients who were followed for an average of 12 months ${ }^{16}$.

Here, we report on the long-term safety and efficacy of high-activity In-111 pentetreotide in 112 patients who received one, two, three or four cycles of $500 \mathrm{mCi}(18.5 \mathrm{GBq})$ of In-111 pentetreotide therapy between Aug 2005 and July 2011.

\section{Materials and Methods}

\section{Patient selection and enrollment}

Patients diagnosed with histopathologically 
confirmed progressive NETs were enrolled in non-randomized trial to evaluate the safety and efficacy of high-dose In-111 pentetreotide therapy in an out-patient setting. This study was performed under approval from the institutional review board at St. Luke's Episcopal hospital, a Baylor College of Medicine affiliated hospital in Texas Medical Center and under an investigational new drug (IND) application from the United States Food and Drug Administration (FDA). All patients gave informed consent prior to treatment.

All patients demonstrated disease progression and had failed routine standard therapy prior to enrollment in the current study. The patients had Karnofsky performance status of higher than 60 . The patients had well differentiated neuroendocrine tumors with or without multiple liver metastases. Inclusion criteria required patients to demonstrate histopathological confirmation of NETs and positive somatostatin receptor scintigraphy. Tumor markers and function were included for diagnosis, such as chromogranin A, serotonin, pancreastatin, gastrin, Neurokinin A, pancreatic polypeptide normetanephrine, alkaline phosphatase (ALP), and 24-h urine 5-hydroxyindole acetic acid (5-HIAA). All included patients had a positive OctreoScan with an uptake grading equal to or exceeding 3 in a 4-point graded scale, also known as Krenning score.

\section{Preparation of High dose In-I I I pentetreotide}

High dose In-111 pentetreotide was prepared as previously described ${ }^{15}$. Indium- 111 chloride ${ }^{111} \mathrm{InCl}_{3}$ ) was purchased from MDS Nordion (Ottawa, ON, Canada), and purified prior to radiolabeling by IsoTex Diagnostics, Inc. (Friendswood, TX). The synthesis of a therapeutic dose of In-111 pentetreotide was obtained by adding ultra-pure ${ }^{111} \mathrm{InCl}_{3}(\sim 500 \mathrm{mCi}(18.5$ GBq)) to 3 standard kits of commercially available Pentetreotide under aseptic conditions. $30 \mu \mathrm{g}$ peptide is used for each therapy.

\section{Treatment Protocol}

Patients were injected with In-111 pentetreotide as an intravenous infusion in physiological saline using a specially designed, lead lined IV pole to decrease radiation exposure to the personnel. The infusion duration was approximately 3 hours, and was performed in an outpatient setting. No amino acid infusion was administered before or after the therapy. Patients were evaluated for adverse events immediately following the therapy and hematological, renal and hepatic toxicities using NCI common toxicity criteria on week 4, 5, 6, and 7 after each cycle of treatment, and every three months thereafter. Patients were eligible for $2^{\text {nd }}$ and $3^{\text {rd }}$ therapy cycles $9-12$ weeks after treatment. The $4^{\text {th }}$ treatment was given approximately 6 months after the third cycle. These patients were evaluated for a clinical and metabolic response prior to each cycle of therapy by interview, completion of quality of life questionnaire and imaging studies, such as CT scan, MRI, Octreoscan and F-18 FDG PET-CT scan.

\section{Dosimetry and biodistribution of In- I I I pen- tetreotide}

In-111 pentetreotide scintigraphy for dosimetry, visualization of tumor spread and assessment of radionuclide uptake intensity was performed. Patients were injected intravenously with 5 to $6 \mathrm{mCi}$ (185-222 $\mathrm{MBq}$ ) of ${ }^{111}$ In-pentetreotide (Mallinckrodt Medical, St. Luis, MO-USA). Static anterior-posterior images were collected, and a single-photon emission computed tomography (SPECT) study was carried out over the regions of tumor using a dual detector gamma scintillation SPECT camera equipped with a medium-energy general purpose collimator (Siemens E-cam). For each patient, whole-body images were performed 10 minutes after injection (before voiding), and at 2, 4, 24, and 48 hours. Regions of interest (ROI) were drawn around the major organs: liver, spleen, kidneys, urinary bladder and whole-body. The data points representing the percentage injected dose (\%ID/organ) were then created and fitted to a mono-exponential, bio-exponential, or an uptake/clearance curve. After curve fitting and integration, the cumulative activity and residence time $(t)$ was calculated for each organ. The femur curve was used to estimate the cumulative activity in the bone marrow. The radiation absorbed dose was calculated by entering the corresponding residence time into the OLINDA software program ${ }^{17}$, which computed the radiation-absorbed dose values as $\mathrm{mGy} / \mathrm{MBq}$ or $(\mathrm{rad} / \mathrm{mCi})$ for each of the target organs.

\section{Statistical analysis}

Kaplan-Meier survival analysis, Log-rank and Wilcoxon tests were performed using Graph-pad Prism 5 software (Graph Pad Software, Inc. La Jolla, CA). All survival times were calculated from the date of the first treatment.

\section{RESULTS}

One hundred and twelve patients (68 male, 44 female) with somatostatin receptor positive NETs underwent treatment with high activity $(500 \mathrm{mCi}$ (18.5 GBq)) In-111 pentetreotide. Of these, 26 patients were treated with one cycle of therapy with an average dose of $490.6 \mathrm{mCi}$ (18.15 GBq), (range 390-503 mCi 
(14.43-18.61 GBq)). Fifty patients received two cycles of therapy with an average dose of $976.6 \mathrm{mCi}(36134.2$ MBq)/patient (range 893-1012 mCi (33-37.4 GBq)) and 28 patients received three cycles of therapy with an average dose of $1461.5 \mathrm{mCi}$ (54 GBq)/patient (range 1419-1495 mCi (52.5-55.3 GBq)). Finally, eight patients received four cycles of therapy with average dose of $1950.5 \mathrm{mCi}$ (72.17 GBq)/patient (1888-2022.54 mCi (69.9-74.8 GBq)). Patient who received one cycle of therapy had an average dose to kidney of $334 \mathrm{rad}$ (range 134-712 rad). Patients who received two cycles of therapy had a cumulative renal dose of $563 \mathrm{rad}$ (range 63-578 rad) and patients who received three treatments had a cumulative renal dose of $654 \mathrm{rad}$ (range 329-2273 rad) and patients who received the four cycles had cumulative average renal dose of 1132 rad (550-1987 rad). Ninty-six patients (85.7\%) had gastroenteropancreatic neuroendocrine tumor (GEP-NET) cancer (67 patients $(59.8 \%$ ) with carcinoid cancer and $29(25.8 \%)$ with pancreatic NET), 5 patients $(4.4 \%)$ had pulmonary NET, 4 patients $(3.6 \%)$ had thymic NET, and the rest of 7 patients $(6.2 \%)$ had other subtypes of neuroendocrine cancer. No significant acute toxicity was observed immediately following treatment and no patients required supportive treatment during therapy. Eleven patients $(9.8 \%)$ had skin rash after treatment and 12 patients $(10.7 \%)$ had mild to moderate nausea/vomiting after their treatment.

Patients were evaluated for evidence of toxicity using NCI common toxicity criteria and followed for an average of 25 months (1.2-56 months, median 18.65 months). Of 92 evaluable patients, 7 patients $(7.6 \%)$ had grade 2 or 3 hematological toxicity, which did not require supportive therapy. Duration of hematological toxicities was 3 weeks (range 1-6 weeks). Grade 2 or 3 liver toxicity was observed in 17 patients $(18.4 \%)$. Patients with neuroendocrine liver metastases who had abnormal liver enzymes and alkaline phosphatase (ALP) levels prior to therapy did not show any significant worsening as a result of the therapy. In addition, 5 patients $(6.1 \%)$ had renal toxicity grade one. Of these patients, 1 patient had received 1 cycle of therapy, 2 patients had received 2 therapies and 2 patients had received 3 therapies. No significant renal toxicity was observed and there was no correlation between renal toxicity and cumulative dose to the kidneys.

Radiological response was initially assessed in 80 evaluable patients for an average of 29 months (1.26-60.2 months) following their last therapy. Analysis was using the Response Evaluation Criteria in Solid Tumors criteria (RECIST criteria). A partial radiological response was observed in 6 patients $(7.5 \%)$. Stable disease was observed in 68 patients $(85 \%)$. The remaining $6(7.5 \%)$ patients had progressive disease.

Hormonal response was assessed in 36 evaluable patients who received three or four cycles of therapy. A biochemical response $(>25 \%$ reduction from pretreatment levels in at least one of the markers (Chromogranin A, Serotonin, 5-HIAA)) was observed in 12 patients (33\%). $32 \%$ of patients had increased levels of all hormonal markers when compared to pre-treatment levels. Statistically, there is no significant difference in tumor marker (Chromogranin A, Serotonin) levels before and after the therapy assays.

Kaplan-Meier survival analysis was conducted on patients who received 2 or more therapies (Fig. 1).

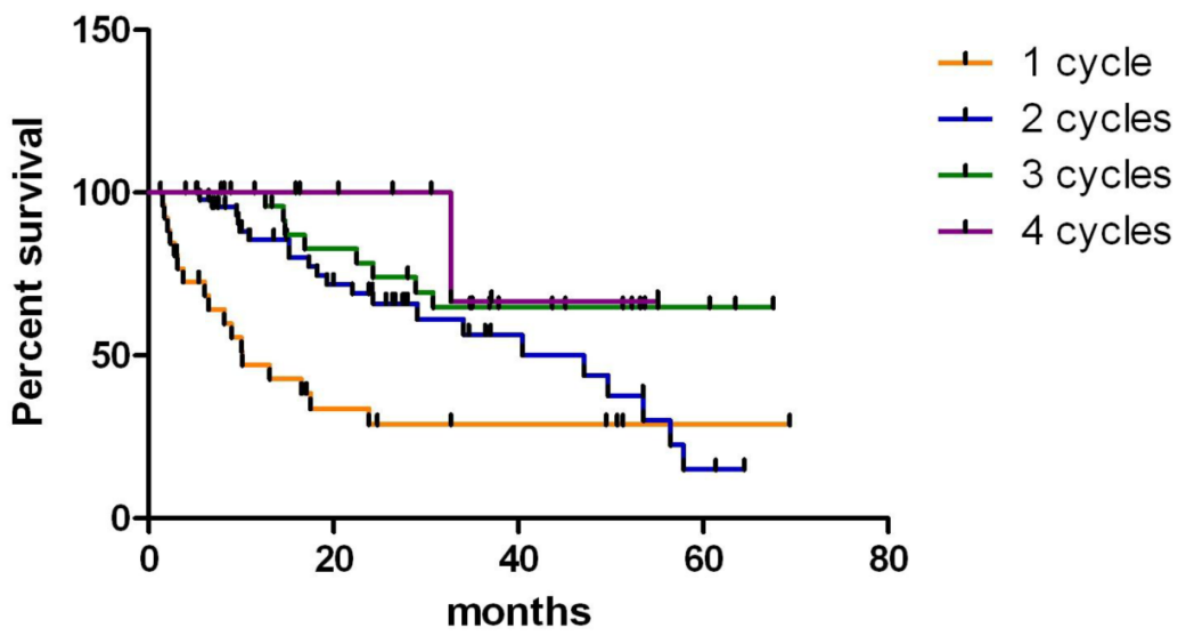

Figure I. Kaplan- Meier survival analysis of neuroendocrine tumor (NET) patients who received one, two, three or four cycles of high activity In-I II pentetreotide therapy. 
The average survival was 24.67 months (after 2 cycles of therapy, 4.97-65.4 months), 30.53 months (after 3 cycles of therapy, 8.83-68.6 months) and 30.19 months (after 4 cycles of therapy, 16.87-56.07 months) (Wilcoxon Test, $\mathrm{P}<0.05,95 \%$ CI 0.5424 to 1.742 ). Two patients with GEP-NET became eligible for curative surgery and both are still alive (24.8 months and 27.37 months following the beginning of therapy). There was no difference between men and women, or ethnic groups. Kaplan-Meier analysis of survival rates among tumor types revealed that patients with islet cell tumors had significantly longer survival times compared to those with carcinoid tumors (Wilcoxon Test, $\mathrm{P}<0.05,95 \%$ CI -0.05838 to 0.8546 ). Median survival for patients with islet cell tumors was 40.77 months compared with median survival time of 16.23 months for patients with carcinoid tumors. There was no significant difference in survival between patients who had unresectable primary tumor with no identifiable metastatic lesions ( $\mathrm{n}=11$, median survival 33.1 months (range 5.40 to 47.3 months)) and metastasis at one site (bone, lung or liver; $\mathrm{n}=52$ median survival 24.6 months (range 8.97 to 54.9 months)). However, survival of patients who had multiple metastatic sties (lung and liver or bone and lung) was significantly decreased compared to those who had metastasis only at one site $(\mathrm{P}<0.01$, Gehan-Breslow-Wilcoxon test, 95\% CI 0.9635-2.215,). Median survival for this group was 16.78 months (range 2.3 to 43.77 months). Average survival of 6 patients with progressive disease was 25.96 months (10.3-62.37 months).

In order to clarify whether there is any correlation between disease burden and survival time, we studied the abdominal CT scan or MRI of 80 evaluable patients who had received at least 2 cycles of therapy and based on the extent of the liver involvement we subcategorized the patients into low bulk, with less than 50\% liver involvement, and high bulk, with higher than $50 \%$ liver involvement. We realized that average survival time of patients $(n=40)$ with high liver involvement after two cycles of therapy is 21.96 months. However, average survival rate in patients $(n=40)$ with lower liver burden is 28.3 months (Log-rank test P $=0.0805,95 \% \mathrm{CI}$ ). Similarly, the survival of patients with less liver involvement who received three or four cycles of therapy is higher, although not statistically significantly, than patients with high liver burden $(\mathrm{P}=0.2754$ and $\mathrm{P}=0.2210$, respectively with $95 \% \mathrm{CI}$ ). These findings suggest that among the patients who have received the same number of therapy cycles, patients with lower liver burden live longer than high bulk group, although this difference is not statistically significant. Importantly, patients who received higher number of therapy could live longer.

Of the 42 patients who had pretreatment PET imaging, 31 patients had positive FDG scans (SUV > 2.5) with an average survival time of 18.9 months (range 1.4- 45.8 months) and 11 patients had negative FDG scans (SUV $\leq 2.5$ ) with an average survival time of 31.8 months (range 7.4- 42.9 months). Survival times for FDG negative patients were significantly longer than those for FDG positive patients $(\mathrm{P}=0.001$ with $95 \%$ confidence).

All patients were asked to complete the functional Living Index questionnaire to assess and compare the quality of life before and after their last cycle of therapy. In patients who received 2 or more cycles of In-111 pentetreotide therapy data showed improvement in social, family, emotional and functional aspects of patient life, particularly following 3 cycles of In-111 PRRT therapy.

\section{DISCUSSION}

Neuroendocrine tumors are a rare, heterogeneous group of malignancies and the incidence of this cancer has significantly increased over the past three decades ${ }^{1,2}$. Typically, these cancers are difficult to diagnose and treat ${ }^{3}$. While surgery can sometimes provide a curative treatment for patients with localized disease, NETs often present as late stage tumors with advanced metastatic disease ${ }^{3}$.

A common feature of differentiated NETs is a high level expression of somatostatin receptors on their cell surface. This feature has been exploited by the use of radio-labeled somatostatin analogues as a tool for diagnosis and treatment for NETS4.

Peptide receptor radionuclide therapy (PRRT) using different radiolabeled somatostatin analogues such as In-111 DTPA-Octreotide, Lu-177 DOTATATE and Y-90 DOTATOC have been used for the treatment of disseminated well differentiated neuroendocrine cancers.

The first clinical trial of In-111 pentetreotide for the treatment of NETs was performed by Krenning et al. ${ }^{7}$ in Netherlands in 1994. Preliminary data from this study demonstrated the safety of repeated treatments with $90-180 \mathrm{mCi}(3.3-6.6 \mathrm{GBq})$ of In-111 pentetreotide administered every 3 weeks for 10 cycles. In this initial study, response correlated with receptor expression. Subsequent studies conducted by the same group confirmed these preliminary findings ${ }^{8}$. Likewise, additional investigational studies have reported significant responses to high-activity In-111 pentetreotide therapy (180-500 $\mathrm{mCi}$ (6.6-18.5 GBq)/treatment) with limited toxicity ${ }^{7-15}$. These studies vary in patient selection, dosing, total activity administered, and reported outcomes, but all show sig- 
nificant benefits and safety of In-111 pentetreotide therapy.

Previously, in a study performed on 26 patients, Anthony et al ${ }^{18}$ reported SD in $81 \%$ of patients along with $\mathrm{CR}+\mathrm{PR}$ equals to $8 \%$, and $12 \%$ of the patients showed progression. In their study, $62 \%$ of the patients experienced symptomatic relief or improvement and median survival was 18 months. Similarly, our prior study ${ }^{16}$ performed on 18 patients showed clinical response in $84 \%$ of the patients, CR + PR equals to $11 \%$ and median survival rate as high as 13.3 months. The results of our current study are consistent with these findings.

Another radiolabeled somatostatin analog that is used for PRRT is Y-90 DOTATOC. Various phase 1 and phase 2 PRRT trials have been performed with this compound ${ }^{17}$. Waldherr et al 20, 21, 22 performed several studies using Y-90 DOTATOC in populations of patients with neuroendocrine cancer. Data from Y-90 DOTATOC studies showed CR + PR rates as high as $24 \%^{20,21}$ and $33 \%^{22}$. However, similar to the data from Anthony et al. ${ }^{18}$, progressive disease was seen in $11 \%^{20,21}$ and $9 \%^{22}$ of patients.

In another study, Kwekkeboom and his colleagues $^{23}$ administered Lu-177 DOTATATE for treatment of 310 patients with neuroendocrine cancer. The data showed CR + PR in $29 \%$ of patients. By including patients with minor response (MR), this number increased to $45 \%$. Stable disease was reported in $35 \%$ of cases.

It is generally accepted that stable disease is the predominant response seen after In-111 pentetreotide therapy ${ }^{16,18}$; although stable disease is still the common outcome after Y-9020, 21, 22 or Lu-17723, 28, 29 therapies, however, partial response rate is generally higher after Y-90 or Lu-177 radionuclide therapies. Nonetheless, in order to study the toxicity profile of patients and to compare the efficacy of each therapy, a randomized clinical trial is needed.

Lu-177 DOTATATE therapies using 4 cycles of $200 \mathrm{mCi}$ (7.4 GBq) Lu-177 DOTATATE and Y-90 DOTATOC using maximum $6 \mathrm{GBq}(160 \mathrm{mCi}) / \mathrm{m}^{28}$ or 7.4 GBq $(200 \mathrm{mCi}) / \mathrm{m}^{29}$ in four cycles produce more cases of PR and CR with requirement of protection of the kidneys using amino acid infusion. Hematological and nephrotoxicity appears to be more common following these therapies while it is rare with In-111 Octreotide therapy.

Recently attempts to improve response to PRRT have included tandem treatments with different radiolabeled analogues. A response to $\mathrm{Lu}-177$ DOTATATE therapy was observed in patients who had previously undergone previous treatment with Y-90 DOTATOC. No additional hematological or re- nal toxicity was observed. Clinical response to Y-90 DOTATOC was predictive of response to Lu-177 DOTATATE24. Retrospective analysis of patients who received up to three cycles of Lu-177 Octreotate following high dose In-111 Pentetreotide therapy showed no significant increase in toxicity ${ }^{25}$, although the effect of tandem therapies on long-term progression free survival remains unclear. As we reported before $^{16}$, due to different peneteration range in tissue, In-111, Y-90 and Lu-177 behave differently once introduced into the tissues. In-111 emits Auger electrons and $\gamma$-rays. The Auger electrons have a maximum penetration range in tissue of $10 \mu \mathrm{m}$. In comparison, Y-90 is a pure $\beta$-emitter with a maximum penetration range in tissue of $12 \mathrm{~mm}$ and $177 \mathrm{Lu}$ is a $\beta$-emitter with a maximum particle range in tissue of $2 \mathrm{~mm}$. Lu-177 also emits $\gamma$-rays, which allows imaging and dosimetry. These differences in particle range may have therapeutic advantages as patients usually have tumors of various sizes.

Additionally, radiosensitizers have been used to improve response to PRRT. The addition of Capecitabine or 5-FU to PRRT has shown no increase in early or late toxicity, and has been shown to improve tumor control and disease stabilization ${ }^{26}$. In terms of the disease burden, there was no significant difference between the number of patients with high bulk liver involvement (50\%) and low bulk patients (50\%).

It is noteworthy that since In-111 is a gamma emitter agent, in order to perform the dosimetry studies and also for imaging purposes, unlike the Y-90- a pure beta emitter-, we do not need a surrogating agent; hence, therapy, dosimetry and imaging is done using the same agent. This is one of the advantages of the in-111 therapy which provides an ease in performing the peptide receptor radionuclide therapy.

Quality of life (QOL) is an important issue in cancer therapy. Previously, Khan et al. ${ }^{27}$ investigated QOL and symptoms after Lu-177 octreotate therapy in patients with inoperable or metastasized gastroenteropancreatic or bronchial neuroendocrine tumors (NETs). The study looked at differences of at least 10 points in global health status (GHS)/QOL scores, symptom scores, and Karnofsky performance scores (KPS) before and after therapy. They realized that regardless of the treatment outcome, patients' quality of life including insomnia, appetite loss, and diarrhea improved significantly. Similarly, Teunissen et al. ${ }^{28}$ evaluated the results of self-assessment questionnaire filled by patients after they received Lu-177 DOTATATE therapy. Kwekkeboom et al 29, 30 showed that other radionuclide materials including Indium-111 and Y-90 are able to improve the QOL of pa- 
tients in a similar manner. Our study revealed significant improvement in social, family, emotional and functional aspects of patient life and supports previous studies.

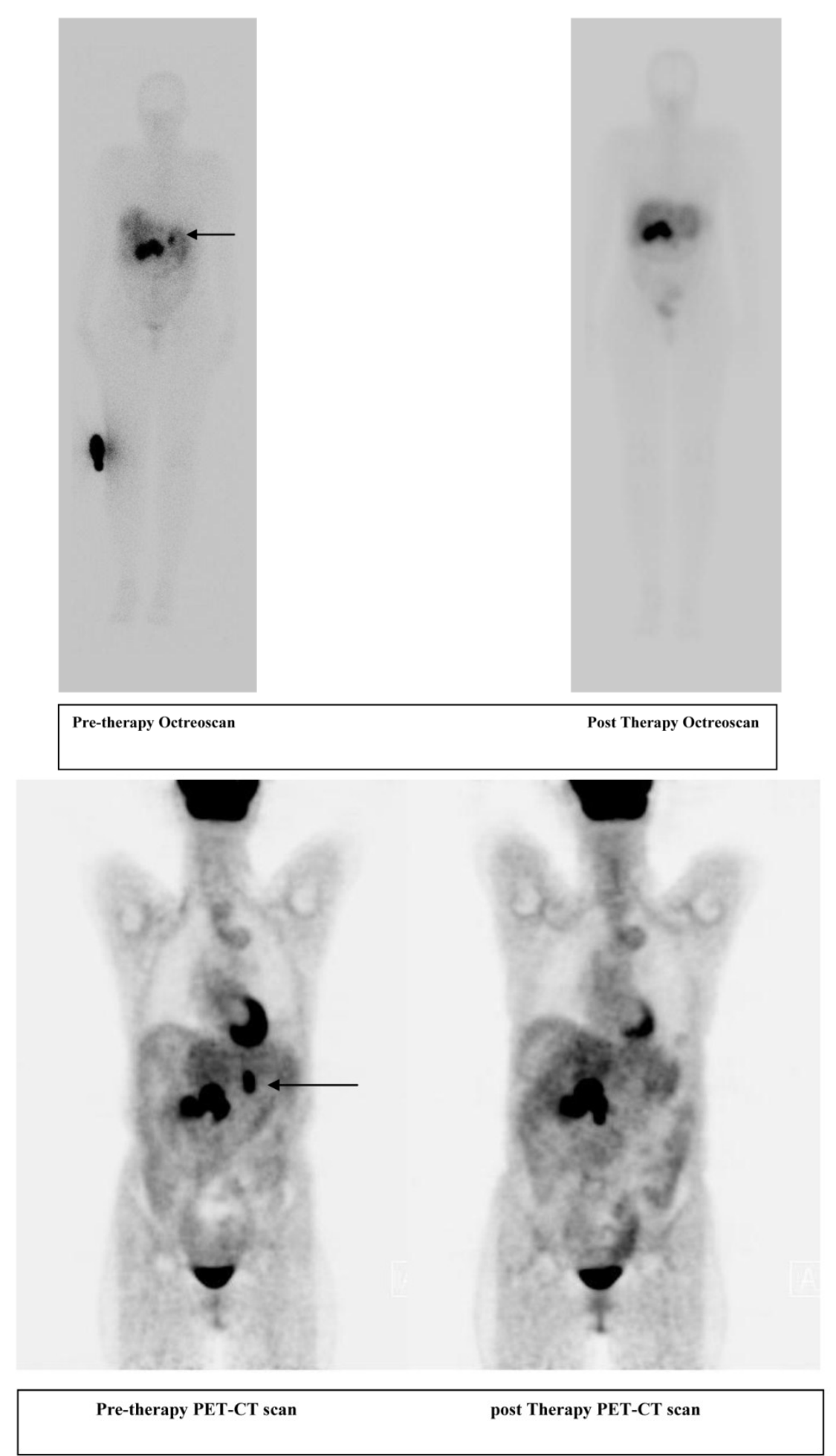

Figure 2. A 56 year old female patient with pancreatic neuroendocrine cancer with partial response to therapy in liver after two cycles of high activity In-I I I Pentetreotide; Octreoscan (top) and F-I8 FDG PET-CT scans (below) comparing pre and post therapy images. 


\section{CONCLUSION}

Our findings demonstrate that high-activity In-111 Pentetreotide therapy is a safe, effective therapy for patients with disseminated neuroendocrine tumors. Our results suggest that the long-term toxicity profile of this agent is excellent, with no major hematological, renal or liver toxicity observed. The absence of renal toxicity at cumulative dose up to 2000 $\mathrm{mCi}(74 \mathrm{GBq})$ suggests that maximal tolerated renal doses have not been reached.

While the observed radiological responses were mostly disease stabilization by the RECIST criteria rather than marked disease regression, this treatment regimen has a clear survival benefit as compared to historical data with limited toxicity. In our study, survival time was increased in patients who received three cycles of therapy compared to those who had two cycles of therapy. The greatest survival times following therapy were observed for patients with islet cell tumors. Tumors originating in the pancreas have been reported to have very high expression of somatostatin receptors and we speculate that this may increase the cellular uptake of the radiotracer and consequently improve the effectiveness of the radiolabeled peptide. Survival times were significantly lower in patients who had multiple sites of metastasis compared to those who had metastasis at only one site. This is most likely due to higher disease burden at the start of treatment and suggests that response rates may be greater for patients with mid-stage disease rather than those with very late stage disease.

There is an increase, although not statistically significant, in survival time in patients with less than $50 \%$ of their liver involved compared to patients with more than half hepatic involvement. Pretreatment FDG status may be a predictor of survival following In-111 pentetreotide therapy.

Hormonal evaluation was performed in patients who had three or more cycles of therapy, but due to a small sample size no statistically significant difference was seen in tumor marker levels before and after the therapy session.

The non-randomized nature of our study is the primary limitation and a randomized clinical trial will be required to determine if multiple cycles of therapy can increase survival times. Moreover, due to the small population, survival advantages for NETs other than carcinoid and islet cell tumors could not be determined.

\section{Competing Interests}

The authors have declared that no competing interest exists.

\section{References}

1. Raut $\mathrm{CP}, \mathrm{Kulke} \mathrm{MH}$, Glickman JN, et al. Carcinoid tumors. Curr Probl Surg. 2006 Jun;43(6):383-450

2. Yao JC, Hassan M, Phan A, et al. One hundred years after "carcinoid": epidemiology of and prognostic factors for neuroendocrine tumors in 35,825 cases in the United States. J Clin Oncol. 2008 Jun 20;26(18):3063-72

3. Taal BG, Visser O. Epidemiology of neuroendocrine tumours. Neuroendocrinology 2004; 80 (Suppl 1):3-7

4. Arnold R, Simon B, Wied M. Treatment of neuroendocrine GEP tumours with somatostatin analogues: a review. Digestion 2000; 62 (Suppl 1):84-91.

5. Prasad V, Baum RP. Biodistribution of the Ga-68 labeled somatostatin analogue DOTA-NOC in patients with neuroendocrine tumors: characterization of uptake in normal organs and tumor lesions. Q J Nucl Med Mol Imaging. 2010 Feb; 54(1):61-7.

6. Stokkel MP, Boot IN, Smit JW. Personal dosimetry of the staff during treatment of neuroendocrine tumours with a high dose of Indium-111 Octreotide. Q J Nucl Med. 2002 Dec; 46(4):331-5.

7. Krenning EP, Kooij PP, Bakker WH, et al. Radiotherapy with a radiolabeled somatostatin analogue, [111InDTPA-D-Phe1]-octreotide. A case history. Ann NY Acad Sci 1994; 733:496-506.

8. Krenning EP, Kooij PP, Pauwels S, et al. Somatostatin receptor: scintigraphy and radionuclide therapy. Digestion. 1996; 57(Suppl 1):57-61.

9. Haselkorn T, Whittemore AS, Lilienfeld DE. Incidence of small bowel cancer in the United States and worldwide: geographic, temporal, and racial differences. Cancer Causes Control 2005; 16:781-7.

10. Buscombe JR, Caplin ME, Hilson AJ. Long-term efficacy of high-activity 111in-pentetreotide therapy in patients with disseminated neuroendocrine tumors. J Nucl Med 2003; 44:1-6.

11. De Jong $\mathrm{M}$, Valkema $\mathrm{R}$, Jamar $\mathrm{F}$, et al. Somatostatin receptor-targeted radionuclide therapy of tumors: preclinical and clinical findings. Semin Nucl Med 2002; 32:133-40.

12. Fjalling $\mathrm{M}$, Andersson $\mathrm{P}$, Forssell-Aronsson E, et al. Systemic radionuclide therapy using indium-111-DTPA-D-Phe1-octreotide in midgut carcinoid syndrome. J Nucl Med 1996;37:1519-21.

13. McCarthy KE, Woltering EA, Espenan GD, et al. In situ radiotherapy with 111In-pentetreotide: initial observations and future directions. Cancer J Sci Am 1998; 4:94-102.

14. Meyers MO, Anthony LB, McCarthy KE, et al. High dose indium 111In pentetreotide radiotherapy for metastatic atypical carcinoid tumor. South Med J 2000; 93: 809-11.

15. Nguyen C, Faraggi M, Giraudet AL, et al. Long-term efficacy of radionuclide therapy in patients with disseminated neuroendocrine tumors uncontrolled by conventional therapy. J Nucl Med 2004; 45:1660-8.

16. Delpassand ES, Sims-Mourtada J, Saso H, et al. Safety and efficacy of radionuclide therapy with high-activity In-111 pentetreotide in patients with progressive neuroendocrine tumors. Cancer Biotherapy $\mathcal{E}$ Radiopharmaceuticals. 2008; 23: 292-300.

17. Stabin MG, Sparks RB, Crowe E. OLINDA/EXM: the second-generation personal computer software for internal dose assessment in nuclear medicine. J Nucl Med 2005; 46:1023-7.

18. Anthony LB, Woltering EA, Espenan GD, et al. Indium-111-pentetreotide prolongs survival in gastroenteropancreatic malignancies. Seminars in Nuclear Medicine. 2002 Apr;32(2):123-32

19. Kwekkeboom DJ, Mueller-Brand J, Paganelli G, et al. Overview of Results of Peptide Receptor Radionuclide Therapy with 3 Radiolabeled Somatostatin Analogs. J Nucl Med 2005; 46:62S-66S.

20. Waldherr $C$, Pless $M$, Maecke HR, et al. The clinical value of [90Y-DOTA]-D-Phe1-Tyr3-octreotide (90Y-DOTATOC) in the treatment of neuroendocrine tumours: a clinical phase II study. Ann Oncol. 2001; 12:941-5.

21. Waldherr $\mathrm{C}$, Pless $\mathrm{M}$, Maecke HR, et al. Tumor response and clinical benefit in neuroendocrine tumors after 7.4 GBq 90Y-DOTATOC. J Nucl Med. 2002; 43:610-6.

22. Waldherr $\mathrm{C}$, Schumacher $\mathrm{T}$, Maecke HR, et al. Does tumor response depend on the number of treatment sessions at constant injected 
dose using 90yttrium-DOTATOC in neuroendocrine tumors? Eur J Nucl Med. 2002; 29 (suppl):S100.

23. Kwekkeboom DJ, de Herder WW, Kam BL, et al: Treatment with the radiolabeled somatostatin analog [177 Lu-DOTA 0 , Tyr3] octreotate: Toxicity, efficacy, and survival. J Clin Oncol 2008 May 1;26(13):2124-30

24. Forrer, F., Uusijarvi, H., Storch, D., et al. Treatment with 177Lu-DOTATOC of patients with relapse of neuroendocrine tumors after treatment with 90Y-DOTATOC. J Nucl Med. 2005 Aug; 46(8):1310-6.

25. Hubble D, Kong G, Michael M et al. 177Lu-octreotate, alone or with radiosensitising chemotherapy, is safe in neuroendocrine tumour patients previously treated with high-activity 111In-octreotide. Eur J Nucl Med Mol Imaging 2010; 37:1869-75.

26. Claringbold PG, Brayshaw PA, Price RA, et al. Phase II study of radiopeptide $177 \mathrm{Lu}$-octreotate and capecitabine therapy of progressive disseminated neuroendocrine tumours. Eur J Nucl Med Mol Imaging, $2011 \mathrm{Feb}$; 38(2):302-11.

27. Khan S, Krenning EP, van Essen $M_{\text {, }}$ et al: Quality of life in 265 patients with gastroenteropancreatic tumours or bronchial carcinoids treated with [177Lu-DOTA0, Tyr3] octreotate. J Nucl Med. 2011 Sep; 52(9):1361-8.

28. Teunissen JJ, Kwekkeboom DJ, Krenning EP: Quality of life in patients with gastroenteropancreatic tumors treated with [177Lu-DOTA0, Tyr3] octreotate. J Clin Oncol 2004 Jul 1;22(13):2724-9.

29. Kwekkeboom DJ, de Herder WW, van Eijck CH, Kam BL, van Essen M, Teunissen JJ, Krenning EP: Peptide receptor radionuclide therapy in patients with gastroenteropancreatic neuroendocrine tumors. Semin Nucl Med 2010 Mar; 40 (2):78-88

30. Kwekkeboom DJ, Kam BL, van Essen M, et al. Somatostatin-receptor-based imaging and therapy of gastroenteropancreatic neuroendocrine tumors. Endocr Relat Cancer. 2010 Jan 29; 17(1):R53-73. 